\title{
What do galaxies look like beyond 31 $\mathrm{mag} / \operatorname{arcsec}^{2}$ ?
}

\author{
María Cebrián ${ }^{1,2}$, Ignacio Trujillo ${ }^{1,2}$ and Juergen Fliri ${ }^{1,2}$ \\ ${ }^{1}$ Instituto de Astrofísica de Canarias, C/Vía Láctea SN, 38205 La Laguna (Tenerife) \\ ${ }^{2}$ Universidad de La Laguna, Dpto. Astrofísica, E-38206 La Laguna, Tenerife, Spain \\ email: mcebrian@iac.es
}

\begin{abstract}
Detection of optical surface brightness structures with magnitudes fainter than 30 $\mathrm{mag} / \operatorname{arcsec}^{2}$ has remained elusive in current photometric deep surveys. We are conducting a new imaging strategy to cross that frontier and address the study of outskirts of galaxies and theories of galaxy formation.
\end{abstract}

Keywords. galaxies: evolution, galaxies: formation, galaxies: halos, techniques: photometric

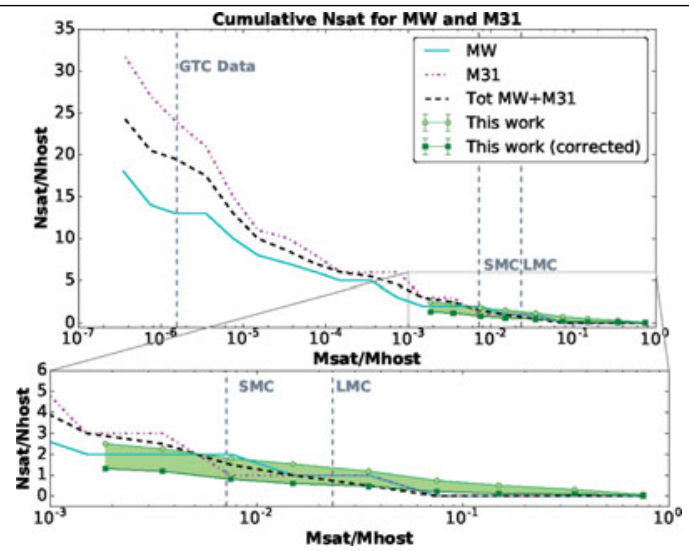

Figure 1. Fraction of satellites per host depending on the mass ratio between the host and the satellite. Green area indicates the average number of satellites found in a spectroscopic SDSS sample of $150 \mathrm{MW}$-like galaxies. Down to $10^{8} \mathrm{M}_{\odot}$ satellites, the abundance of satellites both in M31 and MW are representative of the general population. Upcoming data allow us to explore whether this is also the case down to $10^{5} \mathrm{M}_{\odot}$ satellites (Cebrian \& Trujillo 2016, in prep.).

Cosmological simulations predict a plethora of substructures surrounding galaxies when observed beyond $30 \mathrm{mag} / \operatorname{arcsec}^{2}$ (Cooper et al., 2010). Using the $10.4 \mathrm{~m}$ Gran Telescopio de Canarias telescope and other $\sim 1 \mathrm{~m}$-facilities such as OAJ and LCOGT, we are breaking the $30 \mathrm{mag} / \operatorname{arcsec}^{2}$ frontier. To do this, the flat-fielding and an exquisite treatment of the sky substraction and PSF effects are critical (Trujillo \& Fliri 2015; Fliri \& Trujillo 2016). These data will allow us to study the population of satellite galaxies with a depth comparable to that achieved in the Local Group (see figure 1).

\section{References}

Cooper, A. P., Cole, S., Frenk, C. S., White, S. D. M.., Helly, J., Benson, A. J., De Lucia, G., Helmi, A., Jenkins, A., Navarro, J. F., Springel, V., \& Wang, J. 2010, MNRAS, 46, 744

Fliri, J. \& Trujillo, I. 2016, MNRAS, 456, 1359

Trujillo, I. \& Fliri, J. 2015, ArXiv, 1510.04696 